\title{
Handheld All-In-One Electronics Lab Assistant Device with Usb Connectivity for Engineering Students
}

\author{
D.Helan Helfrish Jency, Dr.S.Sumathi M.E.,Ph.D., \\ PGscholar Department of electronics and communication Adhiyamaan College of engineering, Hosur \\ Head of the Department Department of electronics and communication Adhiyamaan College of Engineering, \\ Hosur
}

\begin{abstract}
Test and measurement instruments such as oscilloscopes and multimeters are the traditional hardware tools. Students in engineering curriculum, when exposed to these instruments, often come across a multitude of equipments such as voltmeters, ammeters, oscilloscopes and even simple things like calculator. Even after the touch screen based smart phone revolution, most of the engineering colleges in India continue the tradition of introducing the students to a decade old instruments. The aim is to design and develop a portable device called Super-Scope which is ALL-IN-ONE electronics lab equipment that has multiple functionalities needed by a modern day engineering student for their practical experiments in electronics and computer labs that would replace the existing plethora of instruments. The device is a fully operated from touch screen using touch buttons and menus.
\end{abstract}

Key words: Oscilloscopes, Multimeters, Super-Scope, Ammeters

\section{INTRODUCTION}

In recent decades, the high cost and administration burdens of physical equipment have caused a significant decline in experimentation within engineering education. This situation has fostered the development and adoption of remote laboratories as a replacement. Recently, remote laboratories based on a large variety of technologies have been developed at multiple universities and adopted in industrial electronics engineering education. In recent years, with the emergence and evolution of communication and Internet technologies, a dramatic shift from traditional learning to online-learning (e-learning) has started to acquire. This shift is more likely to rise, and the way is irreversible owing to the higher efficiency and the unlimited facilities that elearning provides.

The extensive evolutions of the global economy and worldwide competition in the industrial markets have demanded a further restructuring and enhancement of engineering education. So in this, paper Handheld All-in-One Electronics Lab Assistant Device with USB Connectivity has been introduced to overcome from those technologies.

\section{Handheld All-In-One Electronics Lab Assistant Device With Usb Connectivity}

A standpoint advocating that all undergraduate practical experimentation should (or even could) be carried out remotely would be difficult to defend and is not the objective here. Rather, the evaluation of existing implementations has demonstrated that, when used in the right context, remote laboratories can provide significant advantages over conventional proximal laboratories. Whilst there is not yet any significant research data on remote laboratory cost comparisons, anecdotal evidence indicates that operating costs can be significantly reduced. This is in part due to the equipment and apparatus being held in a physically secure environment with tightly con- strained access that limits either intentional or unintentional misuse.

A final advantage which remote laboratories offer is that they present a capability of interinstitutional sharing of laboratory infrastructure and resources. The potential benefits to students are enormous and pro- found, but it requires a global view if it is to be realized .Having accepted that there are considerable "logistic- al" benefits of remote laboratories - flexibility, cost, re- source sharing - attention needs to be given to the impact that a change to remote laboratories has on student learning outcomes. It is clear that the environment in which learning takes place, whether online or face to face, involves a complex array of factors that influence learner satisfaction and achievement. These factors include the relationships between the user and the technology, the instructor and students, and the relationships among the students. This is of particular relevance when considering the evolution of the internet to incorporate increasing support for interactivity and social collaboration.

From a broader perspective, simply referring to the literature to determine an appropriate answer is inconclusive. On the one hand, there is the proposition that there is no significant difference between the educational outcomes from students who performed an experiment remotely, versus those who carried out 
the experiment proximal to the equipment and apparatus. The alternate view how- ever argues that students' performances on different criteria can vary depending upon the form of access used and that indeed some

Out comes appear to be enhanced by non proximal access modes.

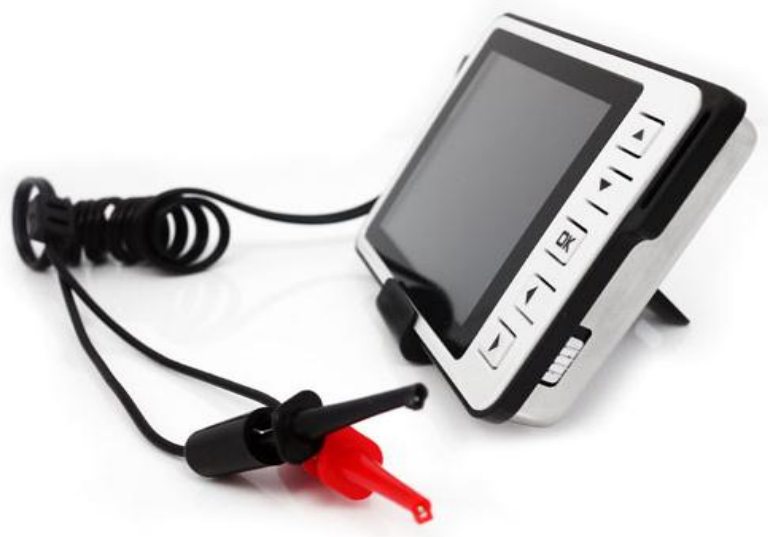

Fig. 1 : Handheld All-in-One Electronics Lab Assistant Device with USB Connectivity

So, having recognized that the nature of the learning outcomes arising from laboratory experiences has a complex relationship with the characteristics of the interaction modality, it is worth considering the way in which the technologies which are used affect the nature of the interaction.

\section{REMOTE LABORATORY ARCHITECTURES}

A design challenge in the practical development of a re- mote laboratory is to identify an architecture which can provide appropriate access to the remote hardware. In the simplest form, the remote laboratory may be a single experiment, with a custom-built web-based interface which may optionally include reporting of measurement data and audio/visual feedback. A more sophisticated facility may involve multiple sets of equipment, multiple experiments and many users. The device has the following features built in:

Digital Signal Oscilloscope - used to monitor signals acquisitioned through the inbuilt 10-bit A to D converter. This device is single channel, $100 \mathrm{KHz}$ bandwidth. The signal s will be shown in color waveforms in a nice $65 \mathrm{~K}$ Color QVGA Touch screen TFT Graphical LCD Display.

- Waveform Storage and Playback - used to save the acquired signals for analyzing and viewing. The storage medium is a 2 GB Micro SD memory card.

- Frequency Generator - used to generate pulses at variable frequencies with added pulse width control

- Logic Analyzer - used to analyze serial protocols such as UART

- Voltmeter - used to measure the input DC voltage

- Ammeter - used to measure the input DC current using current shunt resistor drop

- Ohmmeter - used to find the resistor values, short circuits and components such as diodes

- Tachometer - used to measure the speed of the rotating shaft of the motor using the Rotary Encoder

- Audiometer - used to monitor the audible frequency signals sensed via Microphone circuitry.

- 3-axis Motion Monitor - used to measure acceleration or tilt or motion on all three axis using 3-Axis MEMS Accelerometer

- Light Meter - used to measure the brightness of the incident light in terms of Luminosity using Light Sensor

- Temperature Probe - used to measure the temperature or heat of atmosphere or an object in degree Celsius

- Calculator - used to perform math calculations using touch screen keypad

The software uses Graphics Library and the user interacts with the device using touch screen buttons and menus. The device has a USB-UART bridge circuit that gives USB connectivity for Desktop/Laptop communication for data logging the measured quantities. The logged data can be used for further analysis. This process can be fully controlled by the user from the device UI. It also helps to easily upgrade the firmware of the device from a desktop/Laptop. The device is controlled by LPC1313, a powerful 32-bit ARM Cortex-M3 microcontroller from NxP Semiconductors. 




Fig.2: Architecture

\section{UTILISATION OF TECHNOLOGY AND EDUCATIONAL IMPLICATIONS}

Existing remote laboratory systems utilize the internet in diverse ways. In considering this it is useful to return to the core relationships which exist during student laboratory experimentation. As discussed previously, this includes the relationships between the student and the equipment, between students, and between the instructor and students.

At the core of the interaction between a single student and remote equipment is consideration of the way in which a student engages with that equipment. There are two primary dimensions to this interaction: the extent of the live interactivity, and the richness of the representation of the experimental reality which is exposed to the student. In terms of live interactivity, the iLabs architecture illustrated support for different ends of this spectrum. In batch mode, students submitted their experimental parameters and the experiment request was then queued to be carried out remotely and asynchronously. This form of interaction places relatively low demands on the technology - bandwidth is generally not an issue (there is no requirement for live monitoring) and there is no direct interaction between the student and experiment. Conversely, interactive experiments involve live synchronous interaction with the experiment. Where the monitoring involves video and/or audio, this implies streaming of the media and adaptation of the system to varying bandwidth.

It has long been accepted that peer collaboration can play a major role in affecting student learning outcomes. Also, the majority of conventional (proximal) laboratory exercises are group based (though admittedly this may often have been for logistical rather than pedagogic reasons). Despite this, the vast majorities of current remote laboratories provide limited support for student collaboration, and largely remain one-toone connections between student and equipment. One form of support which is often provided (including in the UTS facilities) is a simple discussion board used separately from the experiment to facilitate student discussion and communication. The advantages of this technology is as follows :

- $\quad$ Replaces the traditionally used lab instruments in an ALL-IN-ONE device

- Enables student to solve problems and experience engineering regardless of where they are - in lecture, in the laboratory, or the study room.

- Enables professors to provide a hands-on learning experience to experiment within the classroom.

- Assists in developing modern engineering systems which involves embedded systems and VLSI design.

- Advanced TFT Color display for rendering clear waveforms and operated with the latest touch screen interface similar to smart phones.

- $\quad$ Designed around LPC1313, a 32-bit ARM Cortex-M3 microcontroller provides high performance at very low power budget suitable for portable equipments like our super-scope.

The UT81B comes in a nice zippered case, with Adapter, banana plug test leads, isolated USB cable, a decent manual, and PC software on CD-ROM. All three of these handheld scopes are intriguing, for different reasons. As a practical, basic instrument that offers ease of use, better bandwidth and accuracy, and durability in the pocket.

The other drawback in the older instruments is its lack of assistance to develop modern engineering systems which involves embedded systems and VLSI design. Embedded Systems and VLSI Design are a culmination of electronics and computers and as a result requires a special understanding of the subject. Embedded system in itself is a very vast field with multiple sub domains and it has already become a basic skill set necessary for a successful career for a graduate in electronics engineering. One of the most relevant problems in designing the laboratory subsystem is remote access to the experiment. The main design objectives taken into account to provide the remote access to the VIs were the following.

* Portability: The visualization environment has to learn hardware platforms and operating systems.

* Usability and accessibility: The visualization and management of an experiment have to be easy to understand and to perform, even for users that are not expert information technologists, and the system 
features have to be accessed easily and homogeneously by students operating at university laboratories or at home.

* Maintenance: The maintenance costs should be reduced to the minimum. This can be made through a Client - server approach that eliminates the need for installing and upgrading application code and data on client computers.

* Client-side common technologies: Students have access to the system using their desktop computers based on common hardware and software technologies, with no need for powerful processors or high memory capacity, and connecting to the Internet through low-speed dial-up connections.

* Security: The remote access of the students through the Internet must preserve the integrity of recorded and transmitted data and of the system as a whole.

* Scalability: System performance must not degrade with the growing number of connected users.

To achieve these objectives, the thin-client model, instead of the classic client-server one, has been chosen. To evaluate the performance of the proposed approach for the remote control of the instrumentation, its capability of providing access to the remote laboratories by means of a common 56-kb/s dial-up connection has been asserted. Many determining factors, in fact, can influence a performance comparison. Some of these are the use of optional mechanisms of the thin-client protocol

(Such as persistent caching and compression for the RDP

Protocol), the application executed on the server, the network

Bandwidth and the kind of traffic that shares the bandwidth

Segment, etc. Ongoing activities focus on the experimental assertion of the robustness of the scheduling system when many systems are being executed contemporarily, and a long request queue should be managed. Moreover, a deep analysis of the security of the proposed thin-client model is being carried out.

\section{Conclusion}

When used appropriately, remote laboratories can provide significant benefits over some proximal laboratories. For these benefits to be realized, consideration must be given to the complex interplay between desired educational outcomes, pedagogical design, and the nature of the technology supporting the laboratory. In this paper the main aim is to design and develop a portable device called Super-Scope which is ALL-IN-ONE electronics lab equipment that has multiple functionalities needed by a modern day engineering student for their practical experiments in electronics and computer labs that would replace the existing plethora of instruments. The device is a fully operated from touch screen using touch buttons and menus.

\section{REFERENCES}

[1]. J. d. J. Rangel-Magdaleno, J. R. Rivera-Guillen, R. d. J. Romero-Troncoso, and R. A. Osornio- Rios. (2011). FPGA-Matlab-based open core for three-time controllers in automatic control applications. Comput. Appl. Eng. Educ. [Online]. Available: http://dx.doi.org/10.1002/ cae.20526.

[2]. E. Monmasson, L. Idkhajine, and M. W. Naouar, "FPGA-based controllers," IEEE Ind. Electron. Mag., vol. 5, no. 1, pp. 14-26, 2011.

[3]. D. V. Pop, M. E. Auer, and D. G. Zutin, "New solution for remote design and test of digital systems with the Altera MAX CPLD Laboratory within the iLab Shared Architecture," in Proc.Remote Eng. Virtual Instrum. (REV) 2011

[4]. Brasov, Romania.

[5]. P. Zenzerovic and V. Sucic, "Remote laboratory for microcontroller systems design," in Proc. Int. Conv. Inf. Commun. Technol., Electron. Microelectron. (MIPRO), 2011, pp. 1685-1688.

[6]. M. Tawfik, E. Sancristobal, S. Martin, C. Gil, P. Losada, G. Diaz, and M. Castro, "Remote laboratories for electrical \& electronic subjects in new engineering grades," in Proc. IEEE Fomento e Innovación con Nuevas Tecnologías en la Docencia de la Ingeniería (FINTDI), 2011, pp. 1-6.

[7]. N. Sousa, G. R. Alves, and M. G. Gericota, “An integrated reusable remote laboratory to complement electronics teaching," IEEE Trans. Learn. Technol., vol. 3, no. 3, pp. 265-271, 2010.

[8]. I. Gustavsson, K. Nilsson, J. Zackrisson, J. Garcia-Zubia, U. Hernandez-Jayo, A. Nafalski, Z. Nedic, O. Gol, J. Machotka, M. I. Pettersson, T. Lago, and L. Hkansson, "On objectives of instructional laboratories, individual assessment,

[9]. and use of collaborative remote laboratories," IEEE Trans. Learn. Technol., vol. 2, no. 4, pp. 263-274, 2009.

[10]. J. E. Corter, J. V. Nickerson, S. K. Esche, C. Chassapis, S. Im, and J. Ma, "Constructing Reality: A Study of Remote,Hands-on and Simulated Laboratories," ACM Transactions onComputer-Human Interaction, vol. 14, 2007.

[11]. S. J. Murray and V. L. Lasky, "A Remotely Accessible Embed- ded Systems Laboratory," in Tools for Teaching Computer Networking and Hardware Concepts, Sarkar, Ed. Hershey: Informa- tion Science Publishing, 2006, pp. $284-302$.

[12]. Massachusetts Institute of Technology. (2008). MIT iCampus: iLabs.[Online].Available:http://icampus.mit.edu/iLabs/default.aspx.

[13]. E. D. Lindsay and M. C. Good, "Effects of laboratory access modes upon learning outcomes," Education, IEEE Transactions on, vol. 48, pp. 619-631, 2005.

[14]. J. E. Corter, J. V. Nickerson, S. K. Esche, and C. Chassapis, "Re- mote Versus Hands-On Labs: A Comparative Study," in34th ASEE/ IEEE Frontiers in Education Conference, Savannah, GA, 2005.

[15]. D. S. Stein and C. E. Wanstreet, "Role of social presence, choice of online or face-to-face group format, and satisfaction with perceived knowledge gained in a distance learning environ- ment," in Midwest Research-to-Practice Conference in Adult, Continuing, and Community Education, Colombus, OH, 2003, pp. 193- 198. enough?," in SEFI 2008: 36th Annual Conference of the European Society for Engineering Education, Aalborg, Denmark, 2008. 
[16]. E. Lindsay, S. Murray, D. Liu, D. Lowe, and C. Bright, "Estab- lishment reality vs maintenance reality: how real is real enough?," European Journal of Engineering Education, (In Press).

[17]. Lindsay, D. Liu, S. Murray, and D. Lowe, "Remote laborato- ries in Engineering Education: Trends in Students' Perceptions," in AaeE 2007: Eighteenth Annual Conference of the Australasian As- sociation for Engineering Education, Melbourne, Australia, 2007, p. (electronic publication).

[18]. H. Mason, in Second Life Education Workshop, Chicago, IL, 2007. 